\title{
Association between polymorphisms in the CYP1A1, CYP2E1 and GSTM1 genes, and smoking, alcohol and upper digestive tract carcinomas in a high-incidence area of northern China
}

\author{
FANG ZHAO $^{1 *}$, JING-FEN SU ${ }^{*}$, SHU-MIN LUN ${ }^{1}$, YONG-JIE HOU ${ }^{1}$, LI-JUAN DUAN ${ }^{1}$, NENG-CHAO WANG ${ }^{2}$, \\ FANG-FANG SHEN ${ }^{3}$, YAO-WEN ZHANG ${ }^{4}$, ZHAO-WEI GAO ${ }^{1}$, JING LI ${ }^{5}$, XIAN-JUAN DU ${ }^{6}$ and FU-YOU ZHOU ${ }^{1}$ \\ ${ }^{1}$ Anyang Key Laboratory for Esophageal Cancer Research and ${ }^{2}$ The Fourth Radiotherapy Department, \\ Anyang Cancer Hospital, Anyang, Henan 455000; ${ }^{3}$ Department of Key Laboratory for Tumor Translational Medicine, \\ The Third Affiliated Hospital, Xinxiang Medical University, Xinxiang, Henan 453000; \\ ${ }^{4}$ The First Radiotherapy Department; ${ }^{5}$ The Third Medicine Department; ${ }^{6}$ The Pathology Department, \\ Anyang Cancer Hospital, Anyang, Henan 455000, P.R. China
}

Received April 26, 2018; Accepted April 14, 2019

DOI: $10.3892 / \mathrm{ol} .2019 .10455$

\begin{abstract}
Metabolic gene variants, smoking, and alcohol consumption are important upper digestive tract cancer (UDTC) risk factors. However, the gene-gene and gene-environment interactions remain unclear. A case-control study in a high incidence area for upper digestive tract cancer was conducted in China. DNA was extracted from buffy coat samples for PCR or PCR-restriction fragment length polymorphism. Smoking and alcohol drinking status was determined by questionnaires. Odds ratios (ORs) and 95\% confidence intervals (CIs) were used to assess the associations. After adjusting for confounding factors, smoking increased esophageal cancer (EC), gastric cardia cancer (GCC) and gastric antral carcinoma (GAC) risk by 3.594, 4.658, and 3.999-fold, respectively. Alcohol consumption increased EC, GCC and GAC risk by $1.953,2.442$ and 1.765 -fold, respectively. The cytochrome P4501A1 (CYP1A1) rs4646903 T>C polymorphism increased GCC risk, the cytochrome P4502E1 (CYP2E1) rs2031920 $\mathrm{C}>\mathrm{T}$ polymorphism increased EC risk, while the GSTM1 null genotype decreased EC risk. An association existed between the following: CYPlA1 rs4646903 and smoking in EC, GCC and GAC; CYPlAl rs4646903 and alcohol consumption in EC and GCC; CYP2E1 rs2031920 and smoking in EC, GCC and GAC and CYP2E1 rs2031920 and alcohol consumption in EC
\end{abstract}

Correspondence to: Professor Fu-You Zhou, Anyang Key Laboratory for Esophageal Cancer Research, Anyang Cancer Hospital, 1 Huanbinbei Road, Anyang, Henan 455000, P.R. China

E-mail: ayzhoufuyou@163.com

${ }^{*}$ Contributed equally

Key words: gene polymorphism, smoking, alcohol drinking, upper digestive cancer, high-incidence area and GCC. No association was observed between $C Y P 1 A 1$ and CYP2E1. The glutathione S-transferase mu 1 (GSTM1) null genotype decreased EC risk $(\mathrm{OR}=0.510)$. Smoking/drinking are upper digestive tract cancer risk factors. The CYPIAl rs4646903 and $C Y P 2 E 1$ rs2031920 polymorphisms were risk factors of GCC or EC, and the GSTM1 null genotype may serve a protective role against EC. The results of the present study indicated that gene-environment interactions increase the risk of UDTC.

\section{Introduction}

Upper digestive tract cancers (UDTC) mainly include esophageal cancer (EC) and gastric cancer (GC). GC can be defined according to the tumor location as proximal or distal gastric adenocarcinoma (1). EC is the eleventh most common cancer and the sixth deadliest cancer worldwide, and GC is ranked fifth for cancer incidence and third for cancer-associated mortalities worldwide (2). Gastric cardia cancer (GCC), or esophagogastric junction cancer, has also become a public health concern (3). To date, several major risk factors have been reported to be associated with UDTC, including heavy smoking and alcohol consumption (4,5). It is widely accepted that the development of UDTC is a result of complex interactions between environmental triggers and genetic factors (6-8). However, these interactions and the exact mechanism of carcinogenesis are still not fully understood.

Metabolites of tobacco and alcohol are first metabolically activated by Phase I enzymes, including cytochrome P4501A1 (CYP1A1) and cytochrome P4502E1 (CYP2E1), into their final forms and then combine with DNA, forming aromatic-DNA adducts that are considered to be an early stage in carcinogenesis (9). These activated forms are subsequently detoxified by Phase II enzymes, particularly GSTM1, a member of the glutathione S-transferases (GSTs) family (10). The CYPlAl rs4646903 $\mathrm{T}>\mathrm{C}$ polymorphism $(\mathrm{MspI})$, also known as the $\mathrm{m} 1$ allele, is a substitution of $\mathrm{T}$ to $\mathrm{C}$ in the non-coding 3'-flanking region which appears to be associated with increased 
enzymatic activity (11). The CYP2E1 rs2031920 C>T polymorphism (RsaI) also known as the c2 allele, involves a $\mathrm{C}$ to $\mathrm{T}$ transition in the 5'-flanking region of the $C Y P 2 E 1$ gene, which appears to be associated with decreased enzymatic activity (12). Individuals who presents the null GSTM1 alleles lack the respective enzyme function (13).

A number of studies have been performed to assess the association between gene polymorphisms and cancer susceptibility (14-18). One meta-analysis showed no association between $C Y P 1 A 1$ rs4646903 polymorphism and digestive tract cancers risk (14), while another meta-analysis confirmed association existed between CYPIA1 rs4646903 and gastric cancer (15). Zhang et al (16) indicated that CYP2E1 rs2031920 polymorphisms revealed no association with the risk of GC, however when GSTM1 was null, the association became significant. GSTM1/T1 null genotype was reported to increase GC risk, and combination of the CYP1A1 rs4646422 variant allele and GSTM1/T1 null genotypes was also associated with a statistically significant increased risk (17). A recent meta-analysis suggested the association between GSTM1 and digestive cancers, and two potential gene-smoking interactions were also found (18). The results from these studies have not always been consistent. In addition, to the best of our knowledge, the evaluation of gene-gene and gene-environment interactions regarding upper digestive cancer risk is insufficient at present. To clarify the combined effects of CYPIAI rs4646903, CYP2E1 rs2031920, GSTM1 null polymorphisms and smoking or alcohol consumption on upper digestive tract cancer risk, a population-based case-control study was performed in Anyang, a typical high-incidence area of upper digestive cancer in Northern China (19,20).

\section{Materials and methods}

Patient and control selection. This case-control study included 194 patients with EC, 212 patients with GCC, 135 patients with gastric antral carcinoma (GAC), and 212 controls. The mean ages \pm standard deviation of these four groups were $63 \pm 7.179$, $64 \pm 9.070,63 \pm 6.852$ and $63 \pm 4.646$ years. The sex ratio (male vs. female) of these four groups were 65.5 vs. $34.5 \%$, 67.9 vs. $32.1 \%, 67.4$ vs. $32.6 \%$ and 66.5 vs. $33.5 \%$. All subjects were recruited from Anyang Cancer Hospital (Henan, China) between July 2015 and July 2017, with the study conducted during the same period. Inclusion criteria were as follows: Age between 30-79 years old with Han ethnicity; pathological diagnosis confirming ECC, GCC or GAC and no simultaneous malignancies. Patients who had undergone chemotherapy or radiotherapy prior to surgery were excluded from the present study. The cancer diagnoses were confirmed histologically. Subjects with no sign of a tumor based on gastroscopy were recruited from a cancer screening program for early detection of upper digestive tract cancers in the same area. All subjects underwent a personal interview and provided information on sociodemographic characteristics, recent and prior tobacco or alcohol use, and family history of cancer. Smoking status was stratified into three levels: Never smoked, smoking for $<30$ years and smoking for $\geq 30$ years; alcohol consumption status was stratified into three levels: Never to occasional; $\geq 1$ day/week and $<150 \mathrm{~g} /$ week; $\geq 1$ day/week and $>150 \mathrm{~g} /$ week. The Anyang Tumor Hospital Institutional Review Board approved the present study. All patients and controls signed a study-specific written informed consent form.

PCR analysis of gene polymorphisms. DNA was extracted from the buffy coat of blood samples from the patients and controls using a FlexiGene DNA kit (cat. no. 51206; Qiagen China Co., Ltd.) for PCR or PCR-restriction fragment length polymorphism (RFLP) experiments. The polymorphisms of CYP2E1 rs2031920 C >T and GSTM1 (21) were detected by PCR using the Thermal Cycler K640 (Hangzhou Jingle Scientific Instrument Co., Ltd.). Nested PCR (22) was used to amplify the CYPlA1 rs4646903 T>C. The PCR thermocycling conditions included initial denaturation at $95^{\circ} \mathrm{C}$ for $15 \mathrm{~min}$ followed by 35 cycles of denaturation at $95^{\circ} \mathrm{C}$ for $1 \mathrm{~min}$, annealing for $1 \mathrm{~min}$ (annealing temperatures are presented in Table I), and extension at $75^{\circ} \mathrm{C}$ for $1 \mathrm{~min}$; and a final extension at $72^{\circ} \mathrm{C}$ for $10 \mathrm{~min}$. The amplified products were digested and examined using $1.5 \%$ agarose gel electrophoresis, and were visualized using a UV transilluminator (Beijing Liuyi Biotechnology Co., Ltd.). Table I presents the primer sequences, annealing temperatures, and digestion enzymes used. A total of $15 \%$ of the PCR products were selected for direct sequencing to confirm the RFLP results. The primers used for $C Y P 1 A 1$ and $C Y P 2 E 1$ sequencing were the same as the primers used in PCR. For GSTM1, the primers used for sequencing were cited from Khabaz et al (23). No deviation was found between the RFLP results and the sequencing data.

Statistical analysis. SPSS 19.0 software (IBM Corp.) was used for statistical analysis, and all tests were repeated three times. Pearson's $\chi^{2}$ test or Fisher's exact test were used to examine differences between groups and unpaired t-tests to compare means. All tests were two-sided. Hardy-Weinberg equilibrium test was used to confirm the CYP1A1 and CYP2E1 genotype distributions. The Bonferroni correction was used to evaluate the associations found and a $\mathrm{P}$-value of $<0.05 / \mathrm{m}$ was considered statistically significant ( $m=$ the total comparison times). Cancer risk associated with genotype or environmental exposure factors was estimated by calculating odds ratios (OR) and 95\% confidence intervals (CI) using unconditional logistic regression. After adjusting for potential confounding factors, multivariate logistic regression was used to assess the association between smoking, alcohol, and the metabolic gene polymorphisms.

\section{Results}

Patient and control characteristics. Table II presents the demographic profiles of the 541 patients and 212 controls. There were no significant differences between the cases and controls in sex, mean age, marital status, education level, labor type and economic income. Upper digestive tract cancer and family history of cancer were significantly associated for EC $(\mathrm{P}=0.017)$, GCC $(\mathrm{P}=0.002)$ and $\mathrm{GAC}(\mathrm{P}=0.001)$.

Detection of CYP1A1, CYP2E1 and GSTM1 variants in upper digestive tract cancers. A total of 194 EC, 212 GCC and 135 GAC cases, and 212 controls were examined to detect $C Y P 1 A 1$ rs4646903, CYP2E1 rs2031920 and GSTM1 polymorphisms. Fig. 1 shows examples of gene polymorphisms in PCR-amplified fragments or digestion fragments. Fig. 2 shows the sequencing 

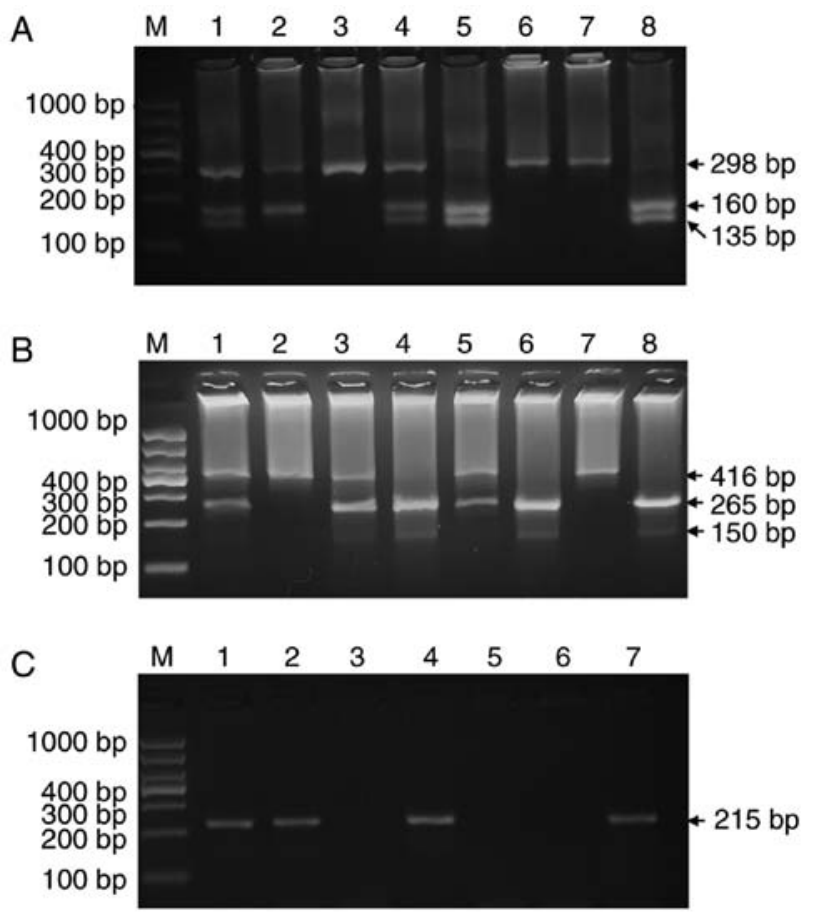

Figure 1. PCR analysis of polymorphisms of CYP1A1 rs4646903, CYP2E1 rs2031920 and GSTM1. (A) CYP1A1 rs4646903 polymorphism: Lane 3, 6 and 7: Wild genotype (298 bp); lane 5 and 8: Homozygous variant (160 and $135 \mathrm{bp}$ ), and lane 1, 2 and 4: Heterozygous variant (298 and $160 \mathrm{bp}$ and 135 bp). (B) CYP2E1 rs2031920 polymorphism: Lane 4, 6 and 8: Wild genotype (416 and 265 and $150 \mathrm{bp}$ ); lane 2 and 7: Homozygous variant (416 bp), and lane 1, 3 and 5: Heterozygous variant (265 and $150 \mathrm{bp).} \mathrm{(C)} \mathrm{GSTM1}$ genotypes: Lane 3,5 and 6: Null genotype (no band) and lane 1, 2, 4 and 7: Wild genotype (215 bp band). CYP1A1, Cytochrome P4501A1; CYP2E1, Cytochrome P4502E1; GSTM1, Glutathione S-transferase mu 1.

chromatogram of CYP1Al rs4646903 and CYP2E1 rs2031920. Among the controls, both the CYP1A1 and CYP2E1 genotype distributions were in Hardy-Weinberg equilibrium.

Association between smoking, alcohol consumption, CYPIAl, CYP2E1, GSTM1 and upper digestive tract cancers. Smoking and alcohol consumption were confirmed to be main risk factors for upper digestive tract cancers (Table III). After adjusting for matching variables and potential confounders, smoking increased EC, GCC and GAC risk compared with non-smoking status: EC [OR $(95 \% \mathrm{CI})=3.594(2.077-6.221)$; $\mathrm{P}<0.001]$; GCC [OR $(95 \% \mathrm{CI})=4.658(2.654-8.174) ; \mathrm{P}<0.001]$ and GAC [OR $(95 \% \mathrm{CI})=3.999(2.131-7.505) ; \mathrm{P}<0.001]$, as $\mathrm{did}$ alcohol consumption: EC [OR $(95 \% \mathrm{CI})=1.953(1.210-3.151)$; $\mathrm{P}=0.006] ;$ GCC $[\mathrm{OR}(95 \% \mathrm{CI})=2.442(1.523-3.914) ; \mathrm{P}<0.001]$ and GAC [OR $(95 \% \mathrm{CI})=1.765(1.030-3.025) ; \mathrm{P}=0.039]$. Dose-dependent trends were observed with these two risk factors, with ORs increasing as the total smoking years or alcohol consumption amount increased (Table III). It was indicated that the GSTM1 null genotype had protective effects against $\mathrm{EC}$, decreasing $\mathrm{EC}$ risk $[\mathrm{OR}(95 \% \mathrm{CI})=0.510$ (0.340-0.765); $\mathrm{P}=0.001]$.

CYP1A1 rs4646903 polymorphism was significantly associated with GCC risk [CC vs. TT: OR $(95 \% \mathrm{CI})=1.936$ $(1.035-3.620), \mathrm{P}=0.039$; $\mathrm{CC}$ vs. $\mathrm{CT}+\mathrm{TT}$ : $\mathrm{OR}(95 \% \mathrm{CI})=2.263$ (1.272-4.026), $\mathrm{P}=0.005$ ]; $C Y P 2 E 1$ rs 2031920 was significantly associated with EC risk [c1/c2 vs. c1/c1: OR $(95 \% \mathrm{CI})=1.673$ 
Table II. Demographic characteristics of patients in the current study.

\begin{tabular}{|c|c|c|c|c|c|c|c|c|c|c|}
\hline \multirow[b]{2}{*}{ Characteristics } & \multirow[b]{2}{*}{ Controls $n=212$} & \multicolumn{3}{|c|}{$\mathrm{EC}$} & \multicolumn{3}{|c|}{ GCC } & \multicolumn{3}{|c|}{ GAC } \\
\hline & & $n=194$ & $\chi^{2}$ & P-value & $\mathrm{n}=212$ & $\chi^{2}$ & P-value & $\mathrm{n}=135$ & $\chi^{2}$ & P-value \\
\hline \multicolumn{11}{|l|}{ Sex } \\
\hline Male & 141 & 127 & 0.049 & 0.824 & 144 & 0.096 & 0.756 & 91 & 0.030 & 0.862 \\
\hline Female & 71 & 67 & & & 68 & & & 44 & & \\
\hline Mean age $\pm \mathrm{SD}^{\mathrm{a}}$, years & $63 \pm 4.646$ & $63 \pm 7.179$ & - & 0.874 & $64 \pm 9.070$ & - & 0.396 & $63 \pm 6.852$ & - & 0.456 \\
\hline \multicolumn{11}{|l|}{ Marital status ${ }^{\mathrm{b}}$} \\
\hline Yes & 209 & 190 & - & 0.836 & 208 & - & 0.685 & 134 & - & 0.147 \\
\hline No & 3 & 4 & & & 4 & & & 1 & & \\
\hline \multicolumn{11}{|l|}{ Education $^{\mathrm{b}}$} \\
\hline$\leq$ Primary school & 136 & 130 & - & 0.320 & 134 & - & 0.974 & 73 & - & 0.127 \\
\hline Junior or senior & 73 & 64 & & & 75 & & & 58 & & \\
\hline$\geq$ College & 3 & 0 & & & 3 & & & 4 & & \\
\hline \multicolumn{11}{|l|}{ Occupation } \\
\hline Labor & 22 & 18 & 3.793 & 0.285 & 25 & 2.567 & 0.463 & 19 & 1.475 & 0.688 \\
\hline Farmers & 175 & 170 & & & 178 & & & 105 & & \\
\hline Civil jobs & 7 & 2 & & & 6 & & & 6 & & \\
\hline Other jobs & 8 & 4 & & & 3 & & & 5 & & \\
\hline \multicolumn{11}{|l|}{ Income $^{\mathrm{c}}$, yuan } \\
\hline$\leq 1,999$ & 130 & 122 & 5.705 & 0.058 & 125 & 0.627 & 0.731 & 76 & 0.939 & 0.625 \\
\hline $2,000-3,999$ & 71 & 70 & & & 78 & & & 52 & & \\
\hline$\geq 4,000$ & 11 & 2 & & & 9 & & & 7 & & \\
\hline \multicolumn{11}{|l|}{ Family history } \\
\hline Yes & 37 & 141 & 5.716 & 0.017 & 64 & 9.475 & 0.002 & 45 & 11.526 & 0.001 \\
\hline No & 175 & 53 & & & 148 & & & 90 & & \\
\hline
\end{tabular}

${ }^{a}$ t-test were used to compare means of age. $\chi^{2}$ test was conducted if the total sample size was $>40$, and the minimum theoretical frequency was $>5$, otherwise, ${ }^{b}$ Fisher's exact probability test was performed. ${ }^{~} \mathrm{RMB}$ per capita/month. CYP1A1, Cytochrome P4501A1; CYP2E1, cytochrome P4502E1; GSTM1, glutathione S-transferase mu 1; EC, esophageal carcinoma; GAC, gastric antral carcinoma; GCC, gastric cardia carcinoma.

$(1.111-2.520), \mathrm{P}=0.014 ; \mathrm{c} 1 / \mathrm{c} 2+\mathrm{c} 2 / \mathrm{c} 2$ vs. $\mathrm{c} 1 / \mathrm{c} 1: \mathrm{OR}(95 \% \mathrm{CI})=1.595$ (1.071-2.375), $\mathrm{P}=0.022$ ] (Tables IV and V).

Gene-gene and gene-environment association between smoking, alcohol consumption, and CYP1A1 or CYP2E1. Gene-gene and gene-environment association between cigarette smoking, alcohol consumption, and CYP1A1 rs4646903 or $C Y P 2 E 1$ rs2031920 polymorphisms are presented in Table VI. An association existed between $C Y P 1 A l$ and smoking in EC, GCC and GAC; $C Y P l A l$ and alcohol drinking in EC and GCC; $C Y P 2 E 1$ and smoking in EC, GCC and GAC; and $C Y P 2 E 1$ and alcohol drinking in EC and GCC. No association was observed between $C Y P 1 A 1$ and $C Y P 2 E 1$. Compared with non-smokers with wild-type CYPlAl (TT), smokers with a $C Y P 1 A 1$ heterozygous variant genotype had a 2.597, 4.359 and 3.503-fold increased risk of EC, GCC and GAC, respectively. Smokers with a CYPlA1 homozygous variant genotype had a 5.125, 8.618 and 6.070-fold increased risk of EC, GCC and GAC, respectively. Compared with non-drinkers with wild-type CYPlAl (TT), alcohol drinkers with a CYPIAl homozygous variant genotype had a 4.124 , 6.820 and 4.489-fold increased risk of EC, GCC and GAC, respectively. Compared with non-smokers with wild-type $C Y P 2 E 1$ (c1/c1), smokers with a CYP2E1 heterozygous variant genotype had a 6.345, 5.318 and 3.300-fold increased risk of EC, GCC and GAC, respectively. In addition, smokers with a $C Y P 2 E 1$ homozygous variant genotype had 6.661 and 7.621-fold increased risk for GCC and GAC. Compared with non-drinkers with wild-type $C Y P 2 E 1(\mathrm{cl} / \mathrm{cl})$, alcohol drinkers with a $C Y P 2 E 1$ heterozygous variant genotype had a 3.820 and 3.070-fold increased risk of EC and GCC, respectively. These results indicated the association between smoking or alcohol consumption and CYP1A1 rs4646903 or CYP2E1 rs2031920 in UDTC. No associations were observed between $C Y P 1 A 1$ rs4646903 and CYP2E1 rs2031920.

\section{Discussion}

In the present study, it was confirmed that smoking and alcohol consumption were the main risk factors of upper digestive tract cancers. In addition, it was indicated that CYP1A1 rs4646903 polymorphisms increased GCC risk, CYP2E1 rs2031920 increased EC risk, while the GSTM1 null genotype decreased EC risk. Regarding the gene-gene 
A

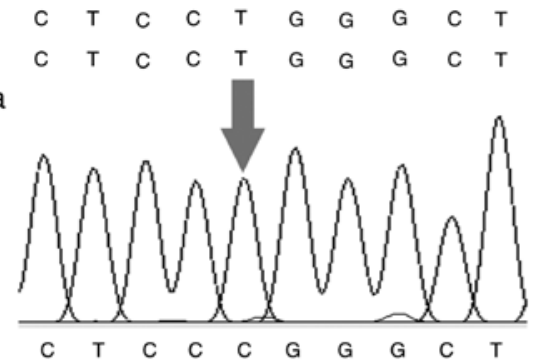

b
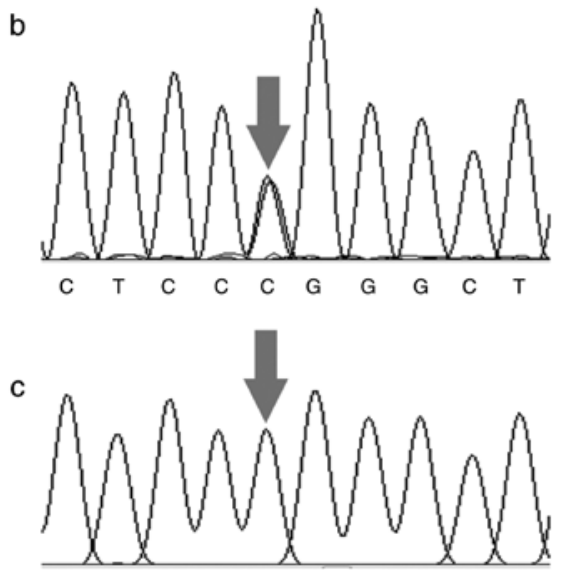

$\begin{array}{lllllllllll}\text { B } & \text { A } & G & \text { T } & \text { A } & \text { C } & \text { A } & \text { A } & \text { A } & \text { A } & \text { T } \\ & \text { A } & \text { G } & \text { T } & \text { A } & \text { C } & \text { A } & \text { A } & \text { A } & \text { A } & \text { T }\end{array}$

a

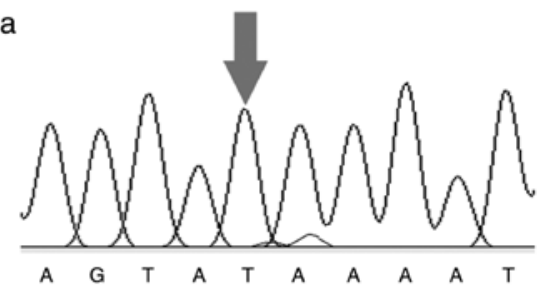

b

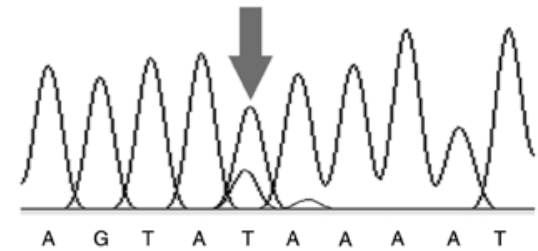

c

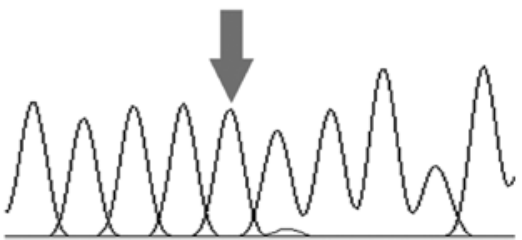

$\mathrm{Ca}$
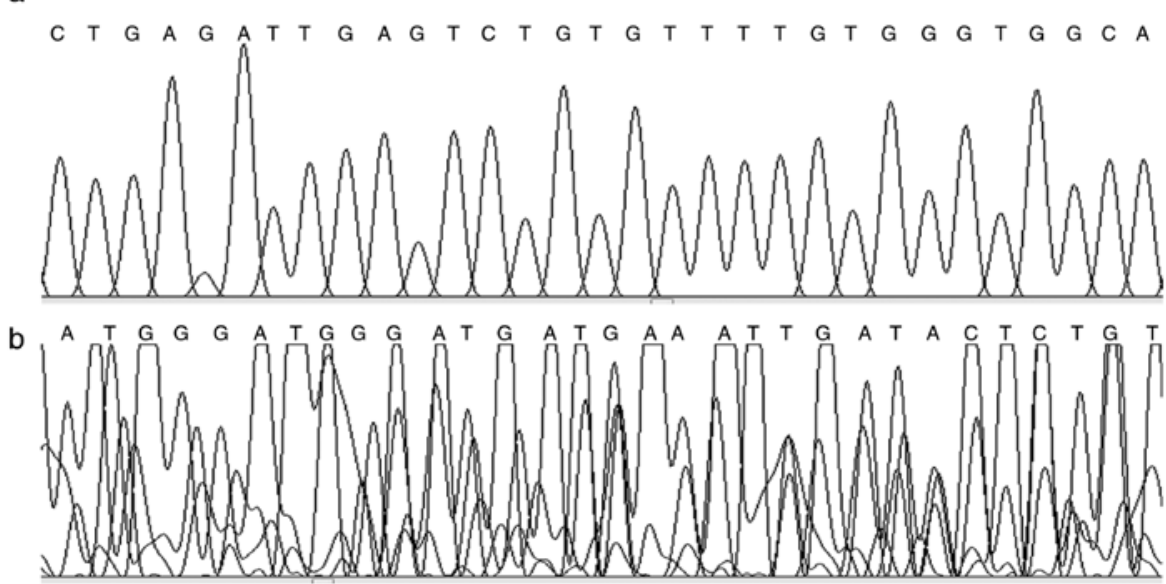

Figure 2. Sequencing chromatogram of CYP1A1 rs4646903, CYP2E1 rs2031920 and GSTM1. (A) Sequencing chromatogram of CYP1A1 rs4646903. The arrow points at the CYP1A1 rs4646903 SNP site. (Aa) Base at the SNP as a T (wild-type homozygous). (Ab) The base to be either a $\mathrm{T}$ or a C (heterozygous T/C). (Ac) Base to be a C (homozygous variant). (B) Sequencing chromatogram of CYP2E1 rs2031920. The arrow points at the CYP2E1 rs2031920 SNP site. (Ba) Base at the SNP as a C (wild-type homozygous). (Bb) Base to be either a $\mathrm{T}$ or a $\mathrm{C}$ (heterozygous $\mathrm{T} / \mathrm{C}$ ). (Bc) Base to be a $\mathrm{T}$ (homozygous variant). (C) Sequencing chromatogram of GSTM1. (Ca) GSTM1 present genotype. (Cb) GSTM1 null genotype. SNP, single nucleotide polymorphism; CYP1A1, Cytochrome P4501A1; CYP2E1, Cytochrome P4502E1; GSTM1, Glutathione S-transferase mu 1.

or gene-environment associations in this study, associations between CYP1A1 rs4646903, CYP2E1 rs2031920 and smoking or alcohol were detected in UDTC.

To date, an increasing number of studies have investigated the associations between CYPIAl rs4646903 polymorphisms and digestive cancer risk $(15,18,24,25)$. In a recent meta-analysis, seven articles reported on CYP1A1 rs4646903 polymorphisms in four digestive cancers, and no associations were found in stratified analysis and subgroup analyses (18). In addition, in another meta-analysis, CYP1A1 rs4646903 polymorphisms were confirmed to be associated with an increased susceptibility to colorectal cancer, however not to esophageal cancer or gastric cancer (24). In the present study, no association between the CYPIA1 rs4646903 CC genotype and EC or GAC were detected, which was consistent with the aforementioned studies. However, in another meta-analysis, 11 studies about CYP1A1 rs4646903 polymorphisms and GC were included, and significant results were found among a large sample-size subgroup (15). Furthermore, evidence was also found to support an association between CYPlAl rs4646903 polymorphisms and digestive tract cancer in the subgroups of Caucasian and mixed individuals (24). This suggested that the associations may vary across different sample sizes and ethnicities. This study found associations between CYP1Al rs4646903 polymorphisms and GCC. To the best of our knowledge, a limited number of studies have been performed in GCC. One report in Linzhou found an association between the CYPIA1 rs4646903 variant allele, and a reduced risk of 


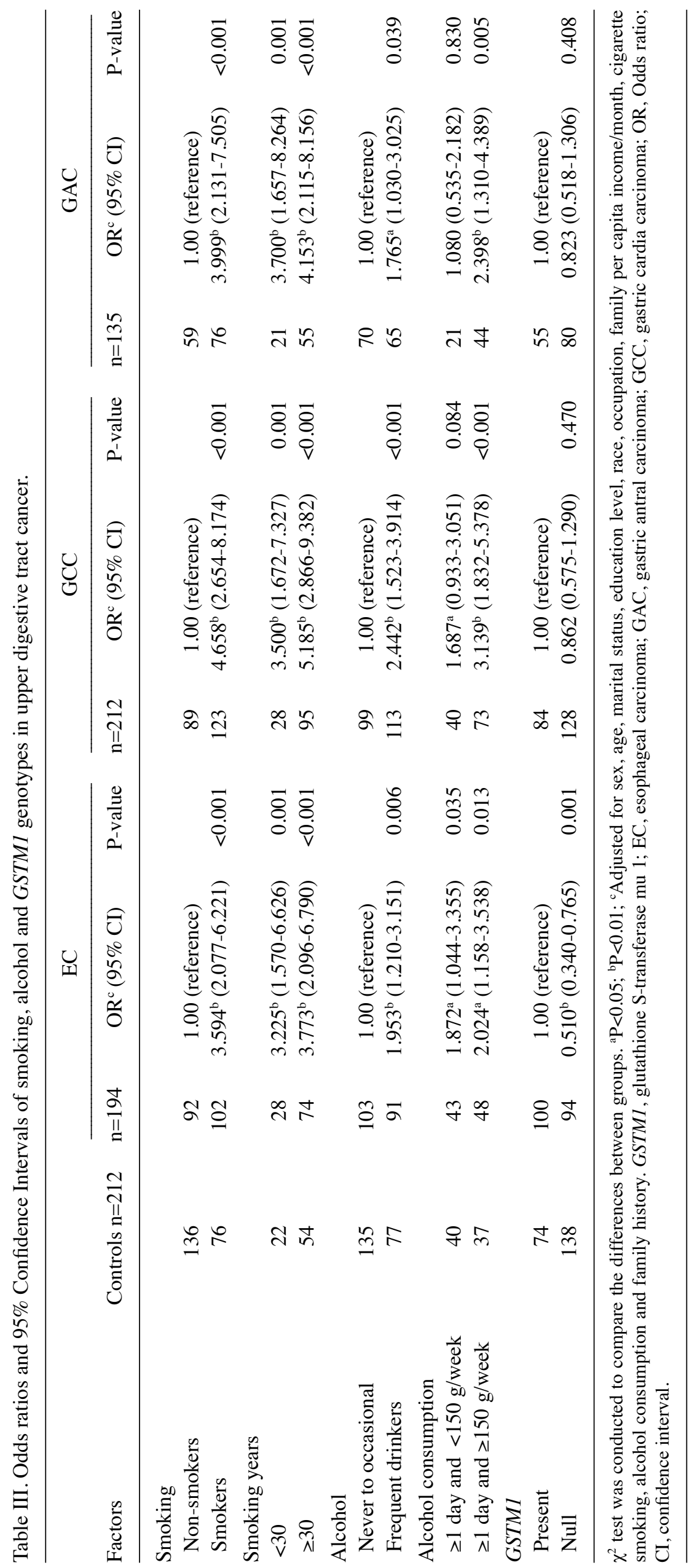



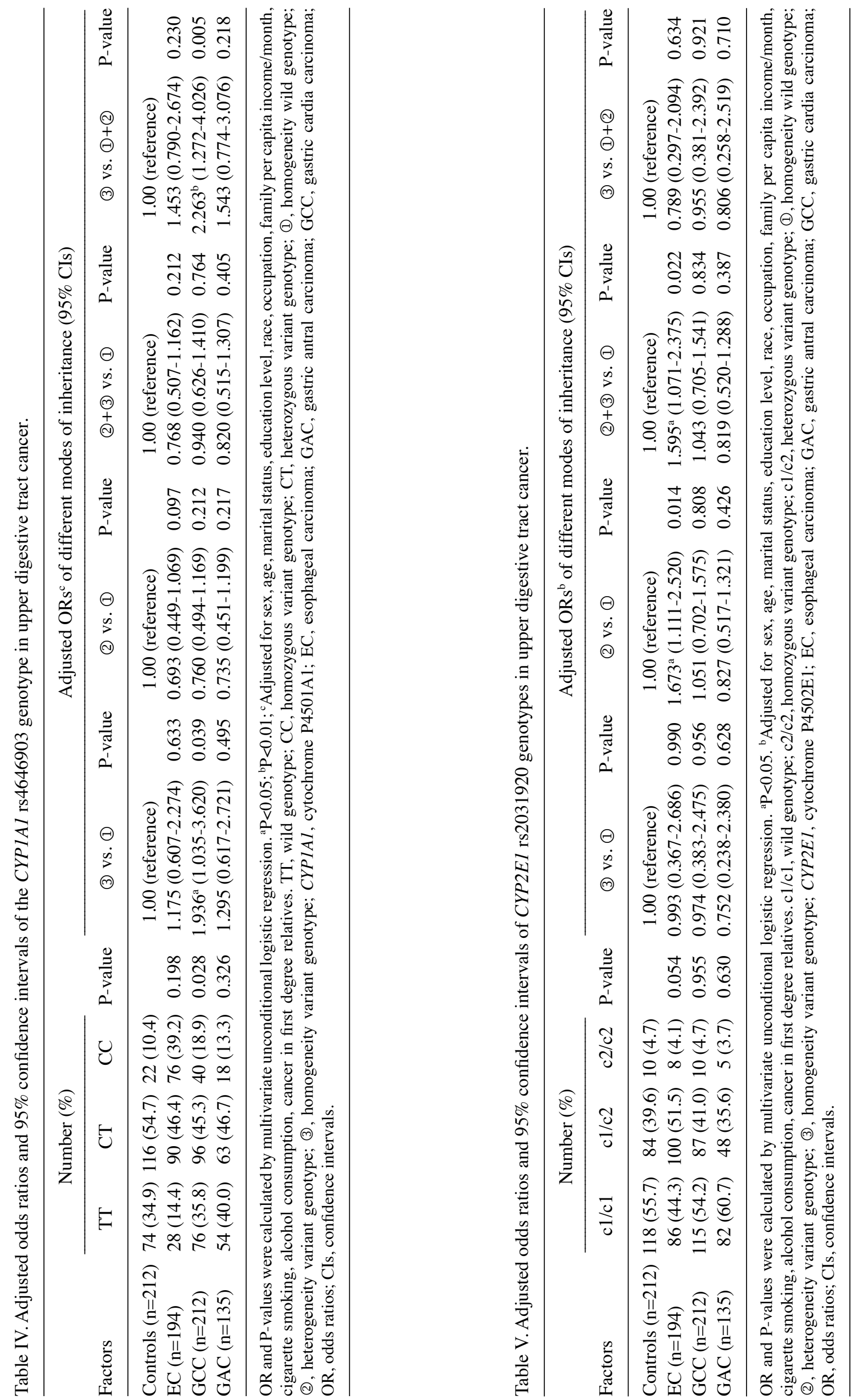


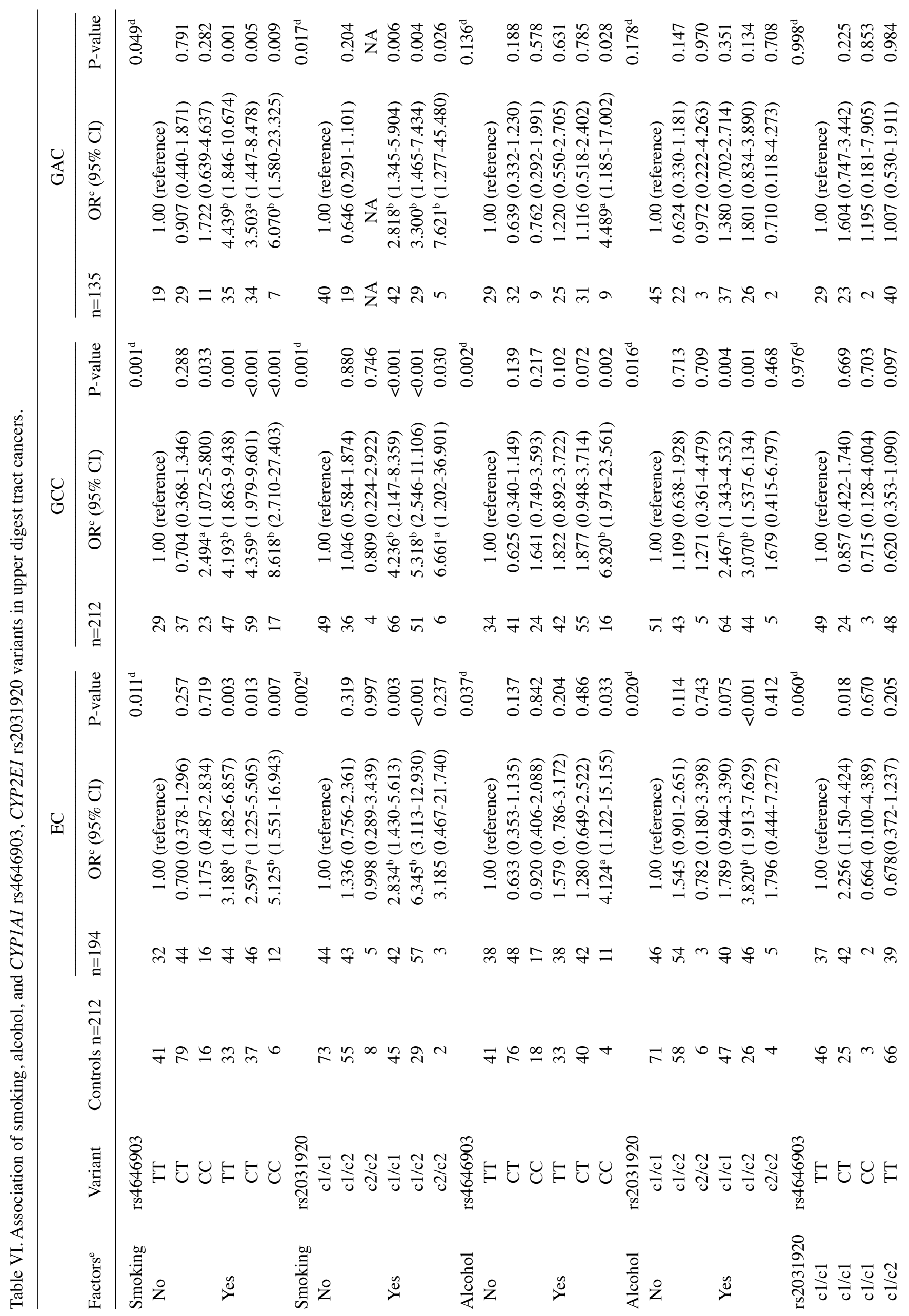


GCC in people with Dysplasia, who were at high risk for the development of GCC (25). However, the study only included 90 cases of GCC, decreasing the reliability of the results.

One meta-analysis in China suggested that the CYP2EI rs2031920 polymorphism was a risk factor for EC, and the c2 allele was demonstrated to be a factor that decreases the risk of EC in the mainland Chinese population (26). However, in this research, CYP2E1 rs2031920 genotypes tended to increase EC risk. One report in Guangzhou Chinese population and another report in a Northern Jiangsu Chinese population also showed that the CYP2E1 rs2031920 polymorphisms could be risk factors for the development of gastric cancer $(27,28)$. Molecular biological evidence has shown that the CYP2E1 rs2031920 variant in the CYP2E1 promoter enhances gene transcriptional activity by altering its binding to its transcription factor, particularly, hepatocyte nuclear factor-1 (29), and influencing its susceptibility to N-nitrosamine-linked carcinogenesis (30), indicating that the CYP2E1 rs2031920 variant may be associated with an increased cancer risk. The present study's results supported the aforementioned findings.

It was indicated that the GSTM1 null genotype had protective effects against EC, decreasing EC risk. However, increased upper digestive tract cancer risk was associated with GSTM1 non-null genotypes. To the best of our knowledge, this is not consistent with most other studies $(17,18)$. A most recent meta-analysis on four digestive cancers showed that the GSTM1 polymorphism was associated with the risk of the four digestive cancers among the Asian population, as subgroup analyses by cancer site showed that the GSTM1 null genotype increased the total gastric cancer risk in the population (18). Another meta-analysis in a Japanese population showed that GSTM1 null, GSTT1 null and GSTM1/T1 both or either null genotypes were associated with increased risk, though this was not statistically significantly (15). However, there are a number of reports showing that cancer risk is associated with GSTM1 non-null genotypes (30-33). There are several possible reasons for this observation. One is that the loss of one GST enzyme may be negligible compared with the large extended GST family (23). Even if the GSTM1 detoxification function is lost, other GST family members can still act to decrease cancer risk. Furthermore, some carcinogens, including $\mathrm{N}$-hydroxy-Trp-P-2, have enhanced genotoxicity and carcinogenicity after binding to glutathione (34). Furthermore, it appears that GSTM1 null individuals have higher DNA adduct levels than GSTM1-expressing individuals (35).

Regarding the gene-gene or gene-environment associations in this study, an association between CYPIAl rs4646903, CYP2E1 rs2031920 and smoking or alcohol was detected. Two meta-analyses showed that CYP2E1 rs2031920 may modify the susceptibility to gastric cancer among individuals who have a smoking history, or when GSTM1 or GSTT1 are null, or CYP2E1 rs2031920 is homozygous wild-type $(16,36)$. An increased risk was seen in CYP1Al rs4646422 variant subjects whose smoking was categorized as $\leq 30$ pack-years, or whose GSTM1/T1 were both null genotypes, or who were null for either GSTM1/T1 individually (17). These studies suggested that tumor incidence is often due to a combination of exposure to external environmental factors and internal gene aberrance. These interactions have a greater impact on cancer susceptibility compared with single genes. 
Associations between metabolic gene polymorphisms and human cancers have been debated. The differences stem from several factors, including ethnic or geographic differences, as Asian populations have been reported to be more prone compared with Caucasian populations to show significant associations between metabolic gene polymorphisms and carcinogenesis $(18,37,38)$. Even in populations containing the same ethnic group, the associations vary by region (14). It is believed that these inconsistent results across ethnicity and geographic areas derive mainly from the unequal frequency of genetic polymorphisms $(30,39)$. Another factor is the different host habits and environmental factor exposure levels, including tobacco use and alcohol consumption $(4,5)$, family history of cancer and Helicobacter pylori infection (40), which have been identified as risk factors for upper digestive tract cancers. Other environmental factors include low socioeconomic status (41), poor oral hygiene (42), nutritional deficiencies, diet (43) and high salt intake (44). It has been hypothesized that various living environments lead to different degrees of cancer susceptibility (45). Specific associations are easily found in subgroups with exposure to negative factors, including smoking, $H$. pylori infection, or low consumption of fruit. A lack of statistical power has also been identified as a contributing factor, as the number of subjects who carry the 'unfavorable' gene polymorphism combinations becomes visible and can be assessed only if sufficient subjects are available with the specific genetic profile required (46). Furthermore, the 'Berkson bias' is typically present in hospital-based studies, as the controls may only represent a sample of an ill-defined reference population and may not be representative of the general population (47). In addition, in terms of gene-gene and gene-environment interaction, tumor incidence is often a combination of multiple factors (48). A negative association between a gene and cancer susceptibility does not mean that the gene has no impact on cancer risk. In terms of methodological differences, the most popular method in previous studies has been PCR-RFLP $(21,30)$. Although PCR-RFLP is a simple, specific and efficient method of SNP detection, it has obvious limitations with respect to accuracy, particularly for subjects who carry a heterozygous mutation (49). With the development of molecular detection technology, a number of researchers have begun to use TaqMan assays $(25,50)$, which may be faster and more accurate compared with PCR-RFLP. A superior new method is genome sequencing $(23,51)$, particularly genome-wide associated studies, which can assay huge amounts of SNPs in a large number of samples and facilitates rapid detection.

In conclusion, it was indicated that smoking/alcohol consumption are upper digestive tract cancer risk factors. The CYP1A1 rs4646903 and CYP2E1 rs2031920 genotypes may contribute to higher GCC and EC susceptibility, respectively. The GSTM1 null genotype may serve a protective effect against EC. The gene-environment associations present increase the cancer risk. In the future, the present study may be improved by increasing the sample size and applying more advanced SNP detection methods, including a TaqMan assay or genome sequencing.

\section{Acknowledgments}

We are also grateful to Dr. Xiang Yuan and Dr Jin-yu Kong at the First Affiliated Hospital of Henan University of Science and Technology University (Xinxiang, China), for their kind assistance in editing this manuscript.

\section{Funding}

This work was supported by grants from the National Natural Science Foundations (grant no. U1504814) and Major Projects of Science and Technology Department in Henan Province (grant nos. 161100311200 and 161100311300) and Xinxiang Science and Technology Project (grant no. CXGG17032).

\section{Availability of data and materials}

The datasets used and/or analyzed during the current study are available from the corresponding author on reasonable request.

\section{Authors' contributions}

FYZ, FZ and SML designed the experiment. FZ, JFS, SML, YJH, LJD, ZWG, JL, XJD, FFS, YWZ and NCW collected the data and performed the experiments. JFS analyzed and interpreted the data. FZ and JFS were major contributors in writing the manuscript. All authors read and approved the final manuscript.

\section{Ethics approval and consent to participate}

The Anyang Tumor Hospital Institutional Review Board approved the present study (no. AZLL022015005150701). All patients and controls signed a study-specific written informed consent form.

\section{Patient consent for publication}

All patients and controls have provided consent for publication.

\section{Competing interests}

The authors declare that they have no competing interests.

\section{References}

1. Piazuelo MB and Correa P: Gastric cáncer: Overview. Colomb Med (Cali) 44: 192-201, 2013.

2. Global Burden of Disease Cancer Collaboration, Fitzmaurice C, Allen C, Barber RM, Barregard L, Bhutta ZA, Brenner H, Dicker DJ, Chimed-Orchir O, Dandona R, et al: Global, regional, and national cancer incidence, mortality, years of life lost, years lived with disability, and disability-adjusted life-years for 32 cancer groups, 1990 to 2015: A systematic analysis for the global burden of disease study. JAMA Oncol 3: 524-548, 2017.

3. Miao Y, Liu R, Pu Y and Yin L: Trends in esophageal and esophagogastric junction cancer research from 2007 to 2016: A bibliometric analysis. Medicine (Baltimore) 96: e6924, 2017.

4. Haas SL, Ye W and Löhr JM: Alcohol consumption and digestive tract cancer. Curr Opin Clin Nutr Metab Care 15: 457-467, 2012.

5. Dong J and Thrift AP: Alcohol, smoking and risk of oesophagogastric cancer. Best Pract Res Clin Gastroenterol 31: 509-517, 2017.

6. Sitarz R, Skierucha M, Mielko J, Offerhaus GJA, Maciejewski R and Polkowski WP: Gastric cancer: Epidemiology, prevention, classification, and treatment. Cancer Manag Res 10: 239-248, 2018.

7. Abnet CC, Arnold M and Wei WQ: Epidemiology of esophageal squamous cell carcinoma. Gastroenterology 154: 360-373, 2018.

8. Matejcic M and Iqbal Parker M: Gene-environment interactions in esophageal cancer. Crit Rev Clin Lab Sci 52: 211-231, 2015. 
9. Miller EC: Some current perspectives on chemical carcinogenesis in humans and experimental animals: Presidential address. Cancer Res 38: 1479-1496, 1978.

10. Kiyohara C, Wakai K, Mikami H, Sido K, Ando M and Ohno Y: Risk modification by CYP1A1 and GSTM1 polymorphisms in the association of environmental tobacco smoke and lung cancer: A case-control study in Japanese nonsmoking women. Int $\mathrm{J}$ Cancer 107: 139-144, 2003.

11. Landi MT, Bertazzi PA, Shields PG, Clark G, Lucier GW, Garte SJ, Cosma G and Caporaso NE: Association between CYP1A1 genotype, mRNA expression and enzymatic activity in humans. Pharmacogenetics 4: 242-246, 1994.

12. Marchand LL, Wilkinson GR and Wilkens LR: Genetic and dietary predictors of CYP2E1 activity: A phenotyping study in Hawaii Japanese using chlorzoxazone. Cancer Epidemiol Biomarkers Prev 8: 495-500, 1999.

13. Strange RC and Fryer AA: The glutathione S-transferases: Influence of polymorphism on cancer susceptibility. IARC Sci Publ: 231-249, 1999.

14. Liu C, Jiang Z, Deng QX and Zhao YN: Meta-analysis of association studies of CYP1A1 genetic polymorphisms with digestive tract cancer susceptibility in Chinese. Asian Pac J Cancer Prev 15: 4689-4695, 2014.

15. Xue H, Lu Y, Xue Z, Lin B, Chen J, Tang F and Huang G: The effect of CYP1A1 and CYP1A2 polymorphisms on gastric cancer risk among different ethnicities: A systematic review and meta-analysis. Tumour Biol 35: 4741-4756, 2014.

16. Zhang MX, Liu K, Wang FG, Wen XW and Song XL: Association between CYP2E1 polymorphisms and risk of gastric cancer: An updated meta-analysis of 32 case-control studies. Mol Clin Oncol 4: 1031-1038, 2016

17. Hidaka A, Sasazuki S, Matsuo K, Ito H, Charvat H, Sawada N, Shimazu T, Yamaji T, Iwasaki M, Inoue M, et al: CYP1A1, GSTM1 and GSTT1 genetic polymorphisms and gastric cancer risk among Japanese: A nested case-control study within a large-scale population-based prospective study. Int J Cancer 139: 759-768, 2016.

18. Du L, Lei L, Zhao X, He H, Chen E, Dong J, Zeng Y and Yang J: The interaction of smoking with gene polymorphisms on four digestive cancers: A systematic review and meta-analysis. J Cancer 9: 1506-1517, 2018

19. Li JY, Liu BQ, Li GY, Chen ZJ, Sun XI and Rong SD: Atlas of cancer mortality in the People's Republic of China. An aid for cancer control and research. Int J Epidemiol 10: 127-133, 1981.

20. Zhou MG, Wang XF, Hu JP, Li GL, Chen WQ, Zhang SW, Wan X, Wang LJ, Xiang C, Hu YS and Yang GH: Geographical distribution of cancer mortality in China, 2004-2005. Zhonghua Yu Fang Yi Xue Za Zhi 44: 303-308, 2010 (In Chinese).

21. Choudhury JH, Singh SA, Kundu S, Choudhury B, Talukdar FR Srivasta S, Laskar RS, Dhar B, Das R, Laskar S, et al: Tobacco carcinogen-metabolizing genes CYP1A1, GSTM1, and GSTT1 polymorphisms and their interaction with tobacco exposure influence the risk of head and neck cancer in Northeast Indian population. Tumour Biol 36: 5773-5783, 2015.

22. Xu X, Kelsey KT, Wiencke JK, Wain JC and Christiani DC: Cytochrome P450 CYP1A1 MspI polymorphism and lung cancer susceptibility. Cancer Epidemiol Biomarkers Prev 5: 687-692, 1996.

23. Khabaz MN, Nedjadi T, Gari MA, Al-Maghrabi JA, Atta HM Bakarman M and Gazzaz ZJ: GSTM1 gene polymorphism and the risk of colorectal cancer in a Saudi Arabian population. Genet Mol Res 15, 2016.

24. Ren A, Qin T, Wang Q, Du H, Zhong D, Hua Y and Zhu L: Cytochrome P450 1A1 gene polymorphisms and digestive tract cancer susceptibility: A meta-analysis. J Cell Mol Med 20: 1620-1631, 2016

25. Roth MJ, Abnet CC, Johnson LL, Mark SD, Dong ZW, Taylor PR, Dawsey SM and Qiao YL: Polymorphic variation of Cyp1A1 is associated with the risk of gastric cardia cancer: A prospective case-cohort study of cytochrome P-450 1A1 and GST enzymes. Cancer Causes Control 15: 1077-1083, 2004.

26. Leng WD, Zeng XT, Chen YJ, Duan XL, Niu YM, Long RP and Luo ZX: Cytochrome P450 2E1 RsaI/PstI polymorphism and risk of esophageal cancer: A meta-analysis of 17 case-control studies. Exp Ther Med 4: 938-948, 2012.

27. Elingarami S, Liu $\mathrm{H}$, Kalinjuma $\mathrm{AV}, \mathrm{Hu} \mathrm{W}, \mathrm{Li} \mathrm{S}$ and $\mathrm{He} \mathrm{N}$ : Polymorphisms in NEIL-2, APE-1, CYP2E1 and mdm2 genes are independent predictors of gastric cancer Risk in a Northern Jiangsu Population (China). J Nanosci Nanotechnol 15: 4815-4828, 2015.
28. Chen ZH, Xian JF and Luo LP: Analysis of ADH1B Arg47His, ALDH2 Glu487Lys, and CYP4502E1 polymorphisms in gastric cancer risk and interaction with environmental factors. Genet Mol Res 15: 2016.

29. Hayashi S, Watanabe J and Kawajiri K: Genetic polymorphisms in the 5'-flanking region change transcriptional regulation of the human cytochrome P450IIE1 gene. J Biochem 110: 559-565, 1991

30. Tan W, Song N, Wang GQ, Liu Q, Tang HJ, Kadlubar FF and Lin DX: Impact of genetic polymorphisms in cytochrome P450 2E1 and glutathione S-transferases M1, T1, and P1 on susceptibility to esophageal cancer among high-risk individuals in China. Cancer Epidemiol Biomarkers Prev 9: 551-556, 2000.

31. Chen C, Madeleine MM, Lubinski C, Weiss NS, Tickman EW and Daling JR: Glutathione S-transferase M1 genotypes and the risk of anal cancer: A population-based case-control study. Cancer Epidemiol Biomarkers Prev 5: 985-991, 1996.

32. Li D, Dandara C and Parker MI: The 341C/T polymorphism in the GSTP1 gene is associated with increased risk of oesophageal cancer. BMC Genet 11: 47, 2010

33. Lewis SJ, Cherry NM, Niven RM, Barber PV and Povey AC: GSTM1, GSTT1 and GSTP1 polymorphisms and lung cancer risk. Cancer Lett 180: 165-171, 2002.

34. Saito K, Yamazoe Y, Kamataki T and Kato R: Glutathione transferase-mediated and non-enzymatic activation and detoxication of the N-hydroxy derivative of Trp-P-2, a potent pyrolysate promutagen. Xenobiotica 14: 545-548, 1984.

35. Houlston RS: Glutathione S-transferase M1 status and lung cancer risk: A meta-analysis. Cancer Epidemiol Biomarkers Prev 8: 675-682, 1999.

36. Zhuo W, Zhang L, Wang Y, Ling J, Zhu B and Chen Z: CYP2E1 RsaI/PstI polymorphism and gastric cancer susceptibility: Meta-analyses based on 24 case-control studies. PLoS One 7: e48265, 2012

37. Wu B, Liu K, Huang H, Yuan J, Yuan W, Wang S, Chen T, Zhao H and Yin C: MspI and Ile462Val polymorphisms in CYP1A1 and overall cancer risk: A meta-analysis. PLoS One 8: e85166, 2013.

38. Meng X, Liu Y and Liu B: Glutathione S-transferase M1 null genotype meta-analysis on gastric cancer risk. Diagn Pathol 9: 122, 2014.

39. Garte S, Gaspari L, Alexandrie AK, Ambrosone C, Autrup H, Autrup JL, Baranova H, Bathum L, Benhamou S, Boffetta P, et al: Metabolic gene polymorphism frequencies in control populations. Cancer Epidemiol Biomarkers Prev 10: 1239-1248, 2001.

40. Choi YJ and Kim N: Gastric cancer and family history. Korean J Intern Med 31: 1042-1053, 2016.

41. Uthman OA, Jadidi E and Moradi T: Socioeconomic position and incidence of gastric cancer: A systematic review and meta-analysis. J Epidemiol Community Health 67: 854-860, 2013.

42. Gupta B and Johnson NW: Emerging and established global life-style risk factors for cancer of the upper aero-digestive tract. Asian Pac J Cancer Prev 15: 5983-5991, 2014.

43. Johnson IT: Understanding the association between diet and nutrition in upper gastrointestinal cancer. Expert Rev Gastroenterol Hepatol 9: 1347-1349, 2015.

44. Goral V: Etiopathogenesis of gastric cancer. Asian Pac J Cancer Prev 17: 2745-2750, 2016.

45. Yoshida T,OnoH,Kuchiba A,Saeki Nand Sakamoto H: Genome-wide germline analyses on cancer susceptibility and GeMDBJ database: Gastric cancer as an example. Cancer Sci 101: 1582-1589, 2010.

46. Taioli E: Gene-environment interaction in tobacco-related cancers. Carcinogenesis 29: 1467-1474, 2008

47. Conn HO, Snyder N and Atterbury CE: The Berkson bias in action. Yale J Biol Med 52: 141-147, 1979.

48. Weinstein IB: Cell culture systems for studying multifactor interactions in carcinogenesis. Dev Toxicol Environ Sci 8: 149-64, 1980.

49. Jin YW, Qu YJ, Wang H, Bai JL and Song F: Limitation of PCR-RFLP method for the detection of genetic mutations in spinal muscular atrophy. Zhonghua Yi Xue Yi Chuan Xue Za Zhi 29: 34-37, 2012 (In Chinese).

50. Blakely T, Barendregt JJ, Foster RH, Hill S, Atkinson J, Sarfati D and Edwards R: The association of active smoking with multiple cancers: National census-cancer registry cohorts with quantitative bias analysis. Cancer Causes Control 24: 1243-1255, 2013.

51. Tan YH, Sidik SM, Syed Husain SN, Lye MS and Chong PP CYP1A1 MspI polymorphism and cervical carcinoma risk in the multi-ethnic population of malaysia: A case-control study. Asian Pac J Cancer Prev 17: 57-64, 2016.

This work is licensed under a Creative Commons Attribution-NonCommercial-NoDerivatives 4.0 International (CC BY-NC-ND 4.0) License. 